\title{
Comparação metalúrgica das ligas semissólidas Al2Si2.5Cu e Al4Si2.5Cu produzidas via processo de extrusão em canal angular
}

\author{
Metallurgical comparison of $\mathrm{Al} 2 \mathrm{Si} 2.5 \mathrm{Cu}$ \\ and Al4Si2.5Cu semisolid alloys produced \\ by equal channel angular pressing process
}

Luis Vanderlei Torres ${ }^{1,2}$, Eugênio José Zoqui ${ }^{2}$

\footnotetext{
${ }^{1}$ Instituto Federal de Educação, Ciência e Tecnologia de São Paulo - IFSP, CEP: 12903-000, Bragança Paulista, SP, Brasil.

${ }^{2}$ Departamento de Engenharia de Manufatura e Materiais, Faculdade de Engenharia Mecânica, Universidade Estadual de Campinas - UNICAMP, CEP: 13083-860, Campinas, SP, Brasil.

e-mail: torres@ifsp.edu.br, torres@fem.unicamp.br, zoqui@ fem.unicamp.br
}

\begin{abstract}
RESUMO
O presente trabalho tem como objetivo contribuir para o desenvolvimento da tecnologia semissólida ao analisar as ligas $\mathrm{Al} 2 \mathrm{Si}$ 2.5Cu e Al4Si2.5Cu produzidas via deformação plástica severa pelo método ECAP (extrusão em canal angular) a fim de compreender o comportamento microestrutural destes materiais. As ligas foram submetidas a tratamentos térmicos de globularização à fração sólida de $60 \%$ e tempo de tratamento de 210 s. A caracterização microestrutural ocorreu via microscopia óptica através da análise de tamanho de glóbulo primário, tamanho de grão, fator de forma de circularidade e RQI (rheocast quality index). As ligas semissólidas processadas via ECAP foram comparadas com as técnicas tradicionais de processamento, a saber, agitação eletromagnética e técnica de ultra-refino de grãos, para a liga $\mathrm{Al} 2 \mathrm{Si} 2.5 \mathrm{Cu}$ não houve redução de tamanho de glóbulos primários e de grãos, porém para a liga Al4Si2.5Cu houve uma redução total de $53 \mu \mathrm{m}$ para o tamanho de glóbulos primários e de aproximadamente $50 \mu \mathrm{m}$ para o tamanho de grãos, característica favorável ao processo de tixoconformação.
\end{abstract}

Palavras-chave: Material semissólido, tixoconformação, processo ECAP, ligas de alumínio.

\section{ABSTRACT}

The present work aims to contribute to the development of semisolid technology by analyzing the $\mathrm{A} 12 \mathrm{Si} 2.5 \mathrm{Cu}$ and $\mathrm{Al} 4 \mathrm{Si} 2.5 \mathrm{Cu}$ alloys produced by severe plastic deformation - ECAP method (equal channel angular pressing) in order to understand the microstructural behavior of these materials. The alloys submitted to reheating treatment in condition of $60 \%$ solid fraction at treatment times of $210 \mathrm{~s}$. Microstructural characterization by optical microscopy through analysis of primary globule size, grain size, circularity shape factor and rheocast quality index (RQI). The semisolid alloys produced by ECAP were compared with the traditional processing techniques, namely electromagnetic stirring and grain ultra-refining technique, the $\mathrm{Al} 2 \mathrm{Si} 2.5 \mathrm{Cu}$ alloy there was no reduction in size of primary globules and grains, but the $\mathrm{A} 14 \mathrm{Si} 2.5 \mathrm{Cu}$ alloy there was a total reduction of $53 \mu \mathrm{m}$ for the primary globules size and approximately $50 \mu \mathrm{m}$ for the grains size, favors characteristic the thixoforming process.

Keywords: Semisolid material, thixoforming, ECAP process, aluminum alloys.

\section{INTRODUÇÃO}

Nos últimos anos, tem-se discutido a necessidade na redução das etapas envolvidas nos processos de fabricação, visando a redução dos custos industriais como também a melhora da qualidade dos produtos obtidos ao final do processo produtivo, sendo assim, a tecnologia semissólida torna-se uma alternativa interessante para a fabricação destes produtos; esta tecnologia baseia-se no processamento de ligas metálicas no estado de coe- 
xistência de fase sólida e fase líquida, produzindo uma estrutura não-dendrítica [1, 2, 3]. Existem diversas rotas de processamento utilizando a tecnologia semissólida e uma das mais promissoras trata-se da deformação plástica severa (DPS). O processamento de metais através da aplicação de deformação plástica severa consiste na aplicação de um elevado grau de deformação plástica ao material, obtendo tamanhos de grãos significativamente menores do que os obtidos em processos tradicionais, ou seja, sendo capaz de gerar tamanhos de grãos em escala sub-micrométrica $(<1 \mu \mathrm{m})$ aumentando a resistência mecânica e sem alterar as dimensões do material $[4,5,6,7]$.

As operações tradicionais de conformação de metais têm sido usadas para a melhoria das propriedades mecânicas e alteração da microestrutura dos metais. Entretanto, as múltiplas reduções da seção transversal do tarugo inicial associadas a estes processos requerem aplicação de cargas muito altas podendo resultar em uma forte heterogeneidade da microestrutura do material ao final do processamento, deficiências estas que os métodos especiais de deformação plástica não possuem [8, 9]. Existem diversos processos de deformação plástica severa como a extrusão em canal angular (ECAP: equal channel angular pressing), torção sob alta pressão (HPT: high-pressure torsion), junção por laminação acumulada (ARB: accumulative roll bonding) e laminação assimétrica (ASR: asymmetric rolling) [10, 11, 12, 13, 14]. O processo de extrusão em canal angular consiste em submeter o material a uma intensa deformação plástica, quando forçado a passar por dois canais de uma matriz que se interceptam formando um ângulo de dobramento $\phi$ (usualmente $90^{\circ}$ ou $120^{\circ}$ ), sob estas condições, o tarugo se move ao longo dos canais e a deformação ocorre por cisalhamento simples [8, 15, 16, 17]. Portanto, este trabalho busca avaliar a tixoconformabilidade das ligas $\mathrm{Al} 2 \mathrm{Si} 2.5 \mathrm{Cu}$ e $\mathrm{Al} 4 \mathrm{Si} 2.5 \mathrm{Cu}$ produzidas via ECAP através de caracterização microestrutural e também contribuir para o desenvolvimento de novas matérias-primas para o processo de tixoconformação.

\section{MATERIAIS E MÉTODOS}

O presente estudo segue a mesma metodologia adotada em trabalhos anteriores $[18,19,20]$, tendo como objetivo gerar e testar novas ligas metálicas para o processo de tixoconformação, a saber, ligas hipoeutéticas de alumínio com menores teores de silício que o convencional através do processo de deformação plástica severa - método ECAP, conforme ilustra a Figura 1.

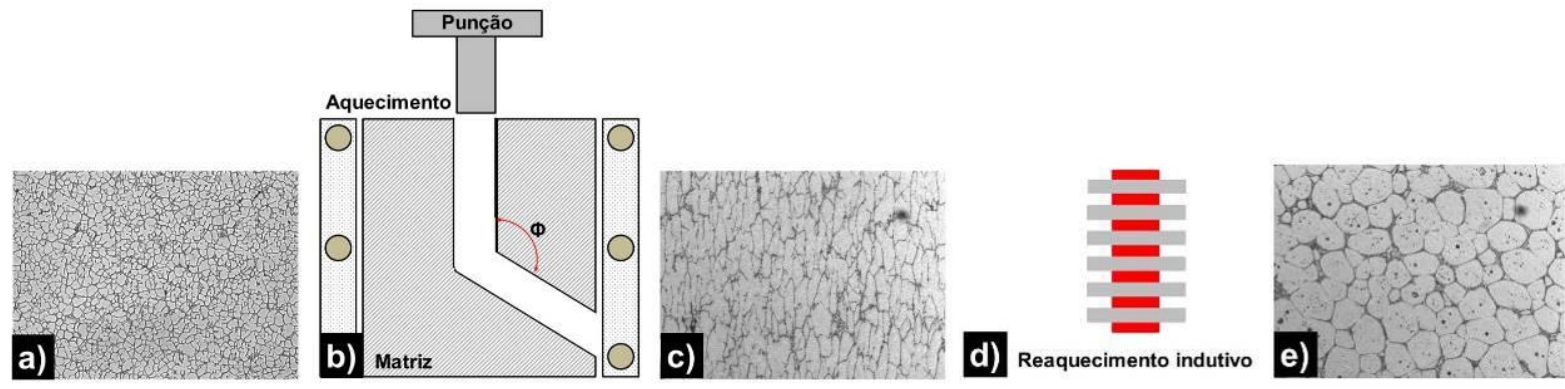

Figura 1: Etapas de processamento da matéria-prima: (a) microestrutura fundida (morfologia de roseta), (b) matriz ECAP, (c) microestrutura fundida + ECAP (morfologia de roseta alongada), (d) reaquecimento indutivo e (e) microestrutura ECAP + reaquecimento (morfologia globular).

\subsection{Produção das ligas}

Antes do início da produção das ligas foram realizadas medições para a determinação do campo eletromagnético efetivo no interior da matriz de solidificação para a potência máxima fornecida pelo sistema de $8 \mathrm{~kW}$, utilizando um medidor de intensidade de campo eletromagnético (gaussímetro), produzindo-se um campo magnético de 13 Gauss. As ligas $\mathrm{Al} 2 \mathrm{Si} 2.5 \mathrm{Cu}$ e $\mathrm{Al} 4 \mathrm{Si} 2.5 \mathrm{Cu}$ foram produzidas via agitação eletromagnética combinada com refinamento de grãos em um forno poço resistivo utilizando-se uma mistura de ligas base, a saber, liga A356, alumínio comercialmente puro, cobre comercialmente puro e refinador de grão Al5Ti1B. Após as ligas atingirem a temperatura de vazamento, ou seja, $50{ }^{\circ} \mathrm{C}$ acima da temperatura liquidus, adicionou-se a liga refinadora com o objetivo de aumentar o efeito da agitação eletromagnética e na sequência o vazamento na matriz de solidificação que estava acoplada ao sistema de agitação eletromagnética, produzindo lingotes com $250 \mathrm{~mm}$ de comprimento e $30 \mathrm{~mm}$ de diâmetro [18,19]; a Tabela 1 apresenta a composição química das ligas obtida por espectrometria de emissão óptica. 
Tabela 1: Composição química das ligas $\mathrm{A} 12 \mathrm{Si} 2.5 \mathrm{Cu}$ e $\mathrm{A} 14 \mathrm{Si} 2.5 \mathrm{Cu}$ [\% peso] obtida por espectrometria de emissão óptica $[18,19]$.

\begin{tabular}{ccccccccc}
\hline Ligas & Si & Cu & Mg & Ti & Fe & Mn & B & Al \\
\hline Al2Si2.5Cu & 2,16 & 2,62 & 0,08 & 0,16 & 0,11 & 0,01 & 0,002 & Balanço \\
\hline Al4Si2.5Cu & 3,84 & 2,54 & 0,18 & 0,15 & 0,14 & 0,01 & 0,002 & Balanço \\
\hline
\end{tabular}

\subsection{Processo ECAP}

A deformação via extrusão por canal angular ocorreu em uma matriz de aço AISI H13 provida de dois canais cilíndricos com diâmetro de $30 \mathrm{~mm}$ que se interceptam formando um ângulo de $120^{\circ}$, utilizando uma prensa hidráulica de 50 toneladas com velocidade máxima de aproximadamente $7 \mathrm{~mm} / \mathrm{s}$, impondo um único passe em lingotes lubrificados com $\mathrm{MoS}_{2}$. Os lingotes de $150 \mathrm{~mm}$ de comprimento e $30 \mathrm{~mm}$ de diâmetro antes de sofrerem a deformação foram aquecidos até a temperatura de $300{ }^{\circ} \mathrm{C}$ para melhorar a passagem do mesmo através da matriz ECAP. A matriz também foi aquecida até a temperatura de $100{ }^{\circ} \mathrm{C}$ para evitar a perda de calor durante o processo [18, 19, 20].

\subsection{Caracterização da temperatura de trabalho}

A determinação da temperatura de trabalho, ou seja, a temperatura para atingir $60 \%$ de fração sólida ocorreu através de simulações via software Thermo-Calc®, sendo realizada com a composição ideal das ligas. As porcentagens de cobre, magnésio, ferro e titânio foram levados em conta, mas elementos residuais, como manganês, cromo, níquel e zinco, não foram; a Tabela 2 apresenta um resumo de todas as temperaturas encontradas.

Tabela 2: Temperaturas obtidas via simulação software Thermo-Calc $[18,19]$.

\begin{tabular}{ccccc}
\hline Ligas & $\mathbf{T}$ solidus $\left[{ }^{\circ} \mathbf{C}\right]$ & $\mathbf{T}$ liquidus $\left[{ }^{\circ} \mathbf{C}\right]$ & $\Delta \mathbf{T}\left[{ }^{\circ} \mathbf{C}\right]$ & T trabalho $\left[{ }^{\circ} \mathbf{C}\right]$ \\
\hline Al2Si2.5Cu & 525 & 639 & 114 & 612 \\
\hline Al4Si2.5Cu & 525 & 627 & 102 & 584 \\
\hline
\end{tabular}

\subsection{Tratamento térmico de globularização}

O tratamento térmico de globularização tem como objetivo conferir a estabilidade microestrutural do material. Assim, as amostras oriundas do processo ECAP com $25 \mathrm{~mm}$ de altura e $30 \mathrm{~mm}$ de diâmetro foram reaquecidas em um forno de indução Norax $(25 \mathrm{~kW}-8 \mathrm{kHz})$ por cerca de 6 minutos à uma taxa de aquecimento de $100{ }^{\circ} \mathrm{C} / \mathrm{min}$. Quando as amostras atingiram as temperaturas equivalentes à fração sólida de $60 \%$ foram mantidas nessas temperaturas pelo tempo de $210 \mathrm{~s}$ e na sequência resfriadas em água. Após o tratamento de reaquecimento, as amostras foram preparadas a partir de técnicas metalográficas para a caracterização microestrutural via microscopia óptica, utilizando metalografia convencional (preto e branco) e metalografia polarizada (colorida); para a metalografia convencional as amostras foram lixadas em lixa d'água até a granulometria de 1500 mesh, polidas e atacadas com ácido fluorídrico ( $1 \mathrm{ml} \mathrm{HF}$ e 99 ml H $\mathrm{H}_{2} \mathrm{O}$ ) com imersão total da amostra por um período de $10 \mathrm{~s}$, posteriormente as mesmas amostras foram atacadas em uma solução eletrolítica de $2 \%$ de $\mathrm{HBF}_{4}$ (ácido fluorbórico) por 6 minutos e tensão de $25 \mathrm{~V}$ para a metalografia polarizada e analisadas em um microscópio óptico Leica DM ILM [18,19].

\section{RESULTADOS E DISCUSSÃO}

As ligas foram caracterizadas microestruturalmente sob as condições: fundida, fundida + ECAP e ECAP + tratada termicamente para $60 \%$ de fração sólida e tempo de tratamento de $210 \mathrm{~s}$. Para as medições do tamanho de glóbulo primário e de grão empregou-se o Método dos Interceptos de Heyn regido pela norma ASTM E112 [21], para o fator de forma de circularidade empregou-se o software de imagens ImageJ $1.40 \mathrm{~g}$ e a partir dos dados de tamanho de glóbulo primário, tamanho de grão e circularidade obtêm-se o RQI (rheocast quality index) através da equação $(\mathrm{RQI}=$ (glóbulo primário $*$ circularidade) $/$ grão) para a qual se espera que o valor encontrado seja o mais próximo de "1", pois nestas condições o tamanho de grão seria igual ao tamanho de glóbulo primário, fato que implica em uma estrutura menos complexa. 

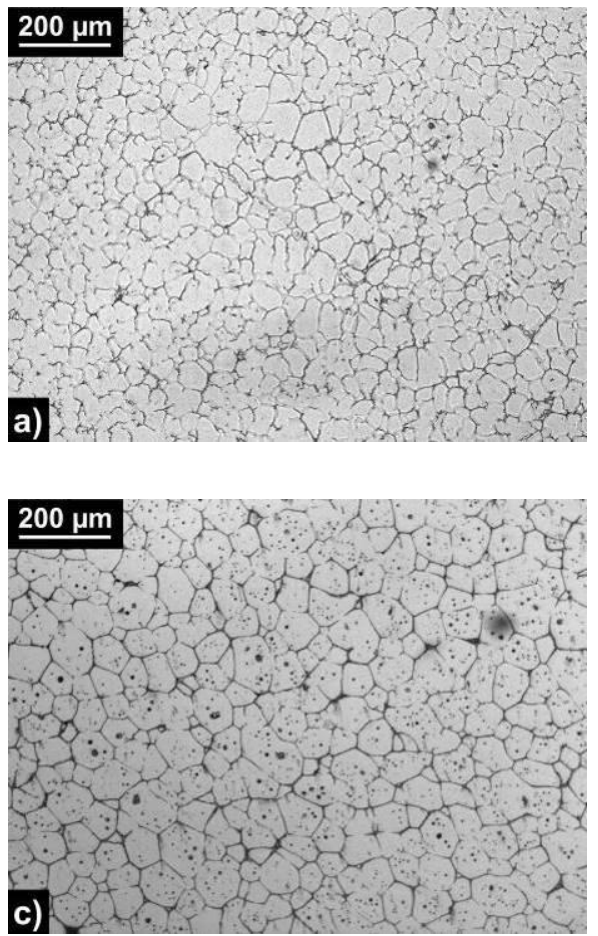
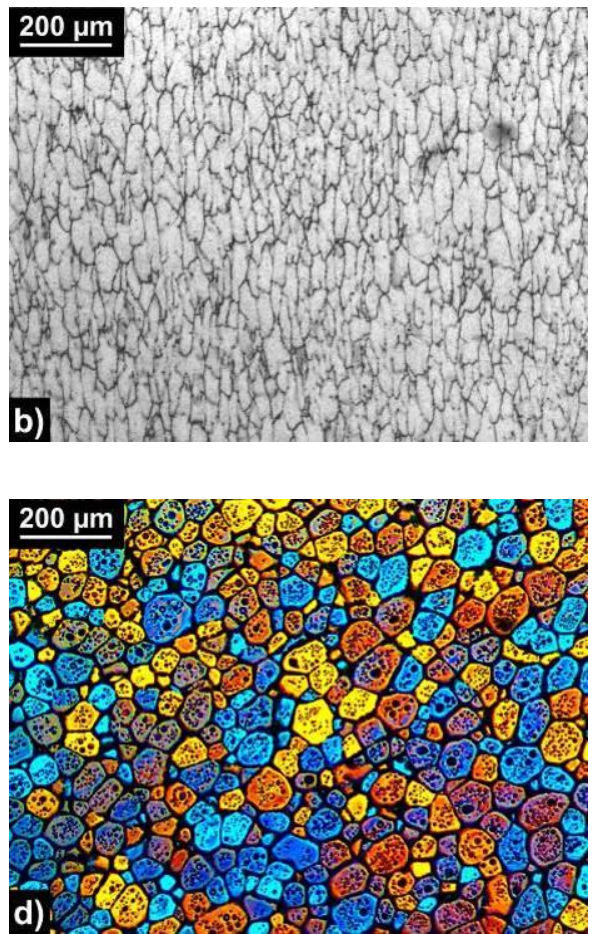

Figura 2: Liga Al2Si2.5Cu - (a) microestrutura fundida, (b) microestrutura fundida + ECAP, (c) microestrutura ECAP + tratada termicamente a $60 \%$ de fração sólida e tempo de $210 \mathrm{~s}$ (metalografias convencionais: atacadas com $1 \%$ de $\mathrm{HF}$ ) e (d) microestrutura ECAP + tratada termicamente a $60 \%$ de fração sólida e tempo de $210 \mathrm{~s}$ (metalografia colorida: atacada com $2 \%$ de $\left.\mathrm{HBF}_{4}\right)$.
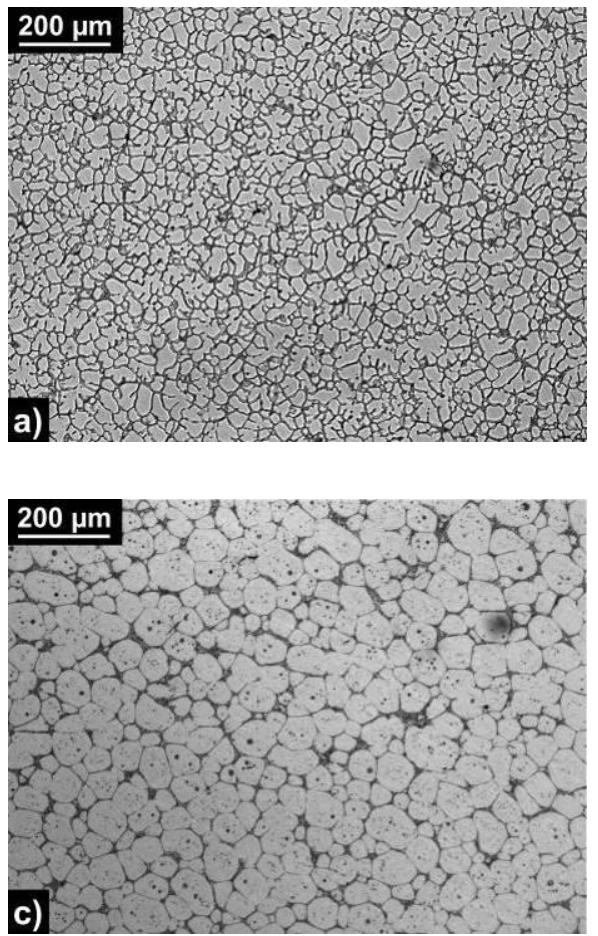
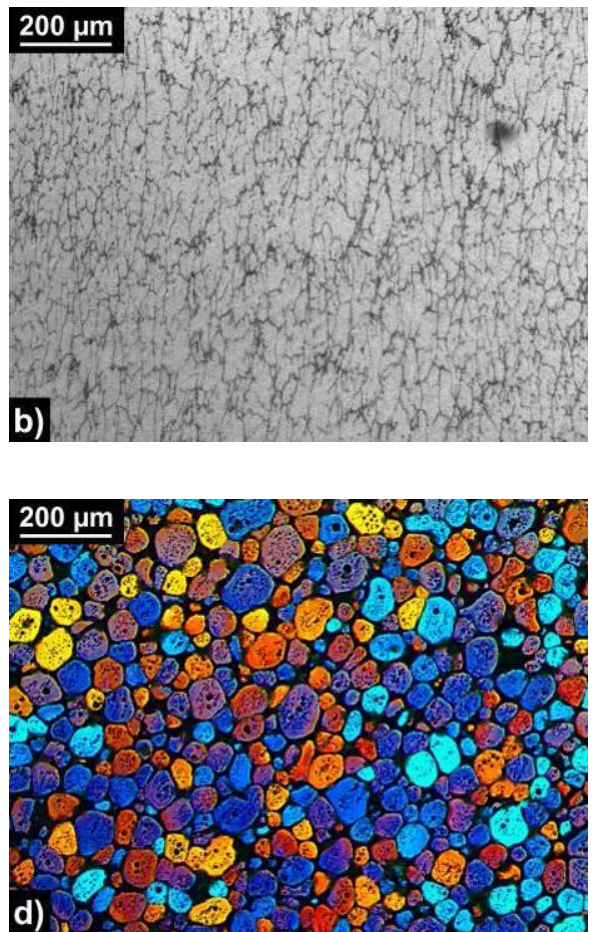

Figura 3: Liga Al4Si2.5Cu - (a) microestrutura fundida, (b) microestrutura fundida + ECAP, (c) microestrutura ECAP + tratada termicamente a $60 \%$ de fração sólida e tempo de 210 s (metalografias convencionais: atacadas com $1 \%$ de HF) e (d) microestrutura ECAP + tratada termicamente a $60 \%$ de fração sólida e tempo de $210 \mathrm{~s}$ (metalografia colorida: atacada com $2 \%$ de $\mathrm{HBF}_{4}$ ). 
Ambas as ligas apresentam uma microestrutura em formato de roseta (microestrutura intermediária entre dendrítica e globular) para a condição fundida, devido ao uso da agitação eletromagnética e à adição da liga refinadora de grãos, resultando em uma microestrutura homogênea conforme visto nas Figuras 2(a) e 3(a) e com formato de rosetas alongadas para a condição ECAP, uma vez que ocorre uma intensa deformação plástica do material conforme visto nas Figuras 2(b) e 3(b). Tem-se que a microestrututra ECAP com apenas um passe teve sua evolução para a morfologia globular, quando reaquecida pelo tempo de tratamento de 210 s e fração sólida de $60 \%$ demonstrando claramente os efeitos de ostwald ripening e coalescência ambos dependentes do tempo de permanência da liga à temperatura de tratamento térmico, conforme visto nas Figuras 2(c), 2(d), 3(c) e 3(d). A matéria-prima quando sofre a deformação durante o processo ECAP, têm-se os grãos alongados e sua energia é armazenada; quando ocorre o reaquecimento da matéria-prima há a recristalização do material, e consequentemente com a energia liberada ocorre a formação de um novo arranjo de cristais, que por sua vez possuem uma morfologia globular [19,20]. Observa-se, que a liga Al2Si2.5Cu quando comparada com a liga A14Si2.5Cu apresenta uma pequena fase eutética nos contornos dos glóbulos primários, devido à menor quantidade de teor de silício em sua composição química, fato também reportado em trabalho anterior [18].

A Tabela 3 apresenta um resumo da caracterização microestrutural para as condições propostas. $\mathrm{O}$ valor do fator de forma de circularidade analisado via metalografia convencional (preto e branco) indica o grau de globularidade da microestrutura e ambas as ligas apresentam uma estrututura globular, com valores próximos a 0,70 uma vez que a escala vai até 1; com relação ao RQI (rheocast quality index) ambas as ligas apresentaram valores satisfatórios, devido ao fato de possuirem pequenos valores de tamanho de glóbulos primários e de grãos com altos valores de cicularidade.

Tabela 3: Valores médios de tamanho de glóbulo primário, circularidade, tamanho de grão e RQI para as condições de ensaio proposta.

\begin{tabular}{ccccc}
\hline Ligas & Glóbulo primário $[\mu \mathrm{m}]$ & Circularidade & Grão $[\mu \mathrm{m}]$ & RQI \\
\hline Al2Si2.5Cu & $60 \pm 7$ & $0,69 \pm 0,12$ & $93 \pm 9$ & $0,44 \pm 0,07$ \\
\hline Al4Si2.5Cu & $49 \pm 6$ & $0,68 \pm 0,13$ & $77 \pm 8$ & $0,43 \pm 0,08$ \\
\hline
\end{tabular}

A Figura 4 apresenta uma comparação das três rotas de processamento mais utilizadas na tecnologia semissólida, a saber: agitação eletromagnética [22], técnica de ultra-refino de grãos [23] e ECAP, para a mesma condição proposta deste estudo, ou seja, fração sólida de $60 \%$ e tempo de tratamento de 210 s. A agitação eletromagnética promove-se no líquido em solidificação pela ação de fortes campos eletromagnéticos, as correntes elétricas induzidas pelo campo promovem uma forte agitação, rompendo a estrutura em formação, estimulando a multiplicação cristalina e sua consequente globularização. Já a técnica de ultra-refino de grãos vem sendo usada comercialmente há mais de 50 anos com agentes nucleantes (liga Al-Ti-B), uma substância adicionada intencionalmente ao líquido para agir como um catalisador da nucleação. Observa-se nas Figuras 4(a) e 4(b) que a liga $\mathrm{Al} 2 \mathrm{Si} 2.5 \mathrm{Cu}$ apresenta valores de tamanho de glóbulos primários e de grãos praticamente idênticos para os processos de agitação eletromagnética e ECAP, somente há uma pequena redução quando comparada com a técnica de ultra-refino de grãos; para a liga $\mathrm{Al} 4 \mathrm{Si} 2.5 \mathrm{Cu}$ ocorre a redução dos valores de tamanho de glóbulos primários e de grãos desde a técnica de ultra-refino de grãos até o processo ECAP, apresentando uma redução total de $53 \mu \mathrm{m}$ para o tamanho médio de glóbulo primário e de aproximadamente $50 \mu \mathrm{m}$ para o tamanho médio de grão. Assim, quando utilizado o processo ECAP para ligas de baixo teor de silício não há uma melhora significativa, porém quando se aumenta o teor de silício das ligas há um melhor refinamento da microestrutura do material, fato observado por Proni et al. [20].

Torres et al. [19] ao analisarem a liga $\mathrm{A} 14 \mathrm{Si} 2.5 \mathrm{Cu}$ em diferentes tempos de tratamentos térmico de globularização obteve valores muito semelhantes aos apresentados neste estudo, observou que a liga possui uma boa estabilidade em relação à morfologia semissólida, ou seja, mesmo em diferentes tempos de tratamento não há variações significativas de tamanho de glóbulos primários como também de grãos, característica favorável ao processo de tixoconformação. 

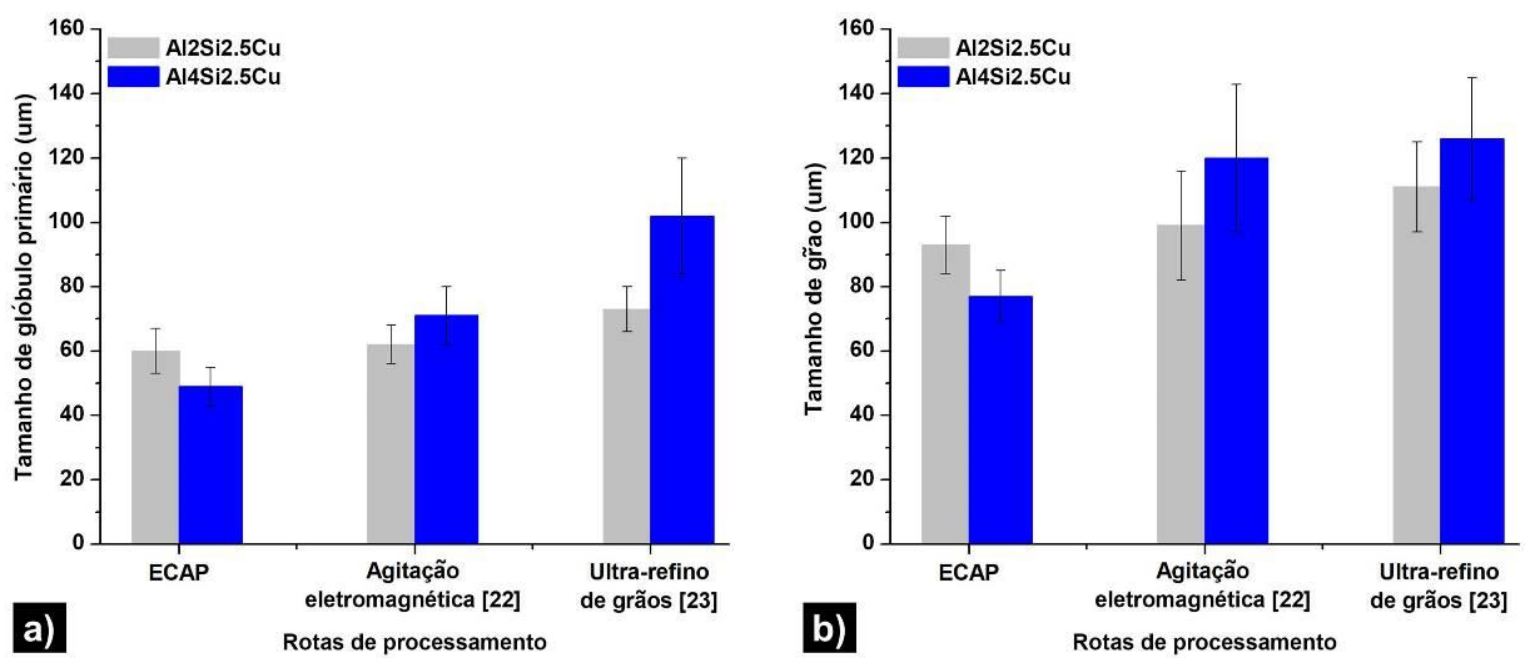

Figura 4: Comparação das diferentes rotas de processamento para as ligas semissólidas $\mathrm{A} 12 \mathrm{Si} 2.5 \mathrm{Cu}$ e $\mathrm{A} 14 \mathrm{Si} 2.5 \mathrm{Cu}$ tratadas termicamente para a fração sólida de $60 \%$ e tempo de tratamento de $210 \mathrm{~s}$ [22, 23].

\section{CONCLUSÕES}

Pode-se concluir que o processo ECAP é adequado à obtenção de matéria-prima para o processo de tixoconformação, uma vez que os resultados demonstram bom comportamento microestrutural, com morfologia globular e bom grau de esfericidade.

\section{AGRADECIMENTOS}

Os autores agradecem a FAPESP (Fundação de Amparo à Pesquisa do Estado de São Paulo - Projeto 2009/08478-1) e a CAPES - Coordenação de Aperfeiçoamento de Pessoal de Nível Superior pelo apoio financeiro, à FEM/UNICAMP - Faculdade de Engenharia Mecânica da Universidade Estadual de Campinas e ao IFSP - Instituto Federal de Educação, Ciência e Tecnologia de São Paulo, campus Bragança Paulista, pelo apoio prático.

\section{BIBLIOGRAFIA}

[1] SPENCER, D. B., MEHRABIAN, R., FLEMINGS, M. C., "Rheological behavior of Sn-15\%Pb in the crystallization range", Metallurgical Transactions, v. 3, pp. 1925-1932, 1972.

[2] FLEMINGS, M. C., RIEK, R. G., YOUNG, K. P., "Rheocasting processes", AFS International Cast Metals Journal, v. 1, pp. 11-22, 1976.

[3] FLEMINGS, M. C., "Behavior of metal alloys in the semi-solid state", Metallurgical Transactions A, v. 22, pp. 957-981, 1991.

[4] VERLINDEN, B., "Severe plastic deformation of metals", Metalurgija - Journal of Metallurgy, v. 11, pp. 165-182, 2005.

[5] VALIEV, R. Z., "Paradoxes of severe plastic deformation", Advanced Engineering Materials, v. 5, pp. 296-300, 2003.

[6] PETRYK, H., STUPKIEWICZ, S., “A quantitative model of grain refinement and strain hardening during severe plastic deformation”, Materials Science and Engineering A, n. 444, pp. 214-219, 2007.

[7] BOWEN, J. R., PRANGNELL, P. B., HUMPHREYS, F. J., "Microstructural evolution during formation of ultrafine grain structures by severe deformation", Materials Science and Technology, v. 16, pp. 1246-1250, 2000.

[8] SEGAL, V. M., “Materials processing by simple shear”, Materials Science and Engineering A, v. 197, pp. 157-164, 1995.

[9] DE OLIVEIRA, L. F., “Aplicação de extrusão angular em canal em materiais metálicos”, Dissertação de M.Sc., Universidade Federal do Rio de Janeiro, Rio de Janeiro, RJ, Brasil, 2004. 
[10] QUARTIERMEISTER, M. V., Estudo da resistência à corrosão de ligas de alumínio com grãos ultrafinos obtidos por deformação plástica severa através do processo ECAP, Dissertação de M.Sc., Universidade Federal de São Carlos, São Carlos, SP, Brasil, 2017.

[11] ESTRIN, Y., VINOGRADOV, A., "Extreme grain refinement by severe plastic deformation: a wealth of challenging science", Acta Materialia, v.61, pp. 782-817, 2013.

[12] AZUSHIMA, A. et al., "Severe plastic deformation (SPD) processes for metals", CIRP Annals - Manufacturing Technology, v. 57, pp. 716-735, 2008.

[13] VALIEV, R. Z., LANGDON, T. G., "Principles of equal channel angular pressing as a tool of grain refinement”, Progress in Materials Science, v.51, pp. 881-981, 2006.

[14] LANGDON, T., "Twenty five years of ultrafine-grained materials: achieving exceptional properties through grain refinement", Acta Materialia, v. 61, pp. 7035-7059, 2013.

[15] IWAHASHI, Y., et al., "Principle of equal-channel angular pressing for the processing of ultra-fine grained materials", Scripta Materialia, v. 35, n. 2, pp. 143-146, 1996.

[16] KOMURA, S., et al., "Optimizing the procedure of equal-channel angular pressing for maximum superplasticity”, Materials Science and Engineering A, v. 297, pp. 111-118, 2001.

[17] KHAN, Z. A., CHAKKINGAL, U., VENUGOPAL, P., "Analysis of forming loads, microstructure development and mechanical property evolution during equal channel angular extrusion of a commercial grade aluminum alloy", Journal of Materials Processing Technology, v. 135, pp. 59-67, 2003.

[18] TORRES, L. V., BENATI, D. M., ZOQUI, E. J., “Morphological evolution of Al-2wt\%Si-2.5wt\%Cu alloy produced by EMS and EMS enhanced by ECAP”, Solid State Phenomena, v. 217-218, pp. 105-110, 2015.

[19] TORRES, L. V., TORRES, L. F., ZOQUI, E. J., "Electromagnetic stirring versus ECAP: morphological comparison of Al-Si-Cu alloys to make the microstructural refinement for use in SSM processing", Advances in Materials Science and Engineering, v. 2016, article ID 9789061, 2016.

[20] PRONI, C. T. W., TORRES, L. V., HAGHAYEGHI, R., ZOQUI, E. J., "ECAP: an alternative route for producing AlSiCu for use in SSM processing”, Materials Characterization, v. 118, pp. 252-262, 2016.

[21] AMERICAN SOCIETY FOR TESTING AND MATERIALS, ASTM. E112: Standard test methods for determining average grain size, West Conshohocken, 1996.

[22] TORRES, L. V., Tixoconformação de novas ligas Al-Si-Cu, Tese de D.Sc., FEM/UNICAMP, Campinas, SP, Brasil, 2013.

[23] BENATI, D. M., Avaliação da tixoconformabilidade de ligas Al-Xwt\%Si-2,5wt\%Cu-0,5wt\%Mg, Dissertação de M.Sc., FEM/UNICAMP, Campinas, SP, Brasil, 2008.

\section{ORCID}

Luis Vanderlei Torres

Eugênio José Zoqui
http://orcid.org/0000-0002-2249-668X

https://orcid.org/0000-0002-3614-0051 